\title{
Crack initiation in metallic glasses under nanoindentation
}

\author{
Yongjian Yang ${ }^{1,2}$, Jian Luo ${ }^{1}$, Liping Huang ${ }^{1,2}$, Guangli Hu${ }^{1}$, K. Deenamma Vargheese ${ }^{1}$, \\ Yunfeng Shi ${ }^{1,2 *}$, John C. Mauro ${ }^{1 *}$ \\ ${ }^{1}$ Science and Technology Division, Corning Incorporated, Corning, New York 14831, USA \\ ${ }^{2}$ Department of Materials Science and Engineering, Rensselaer Polytechnic Institute, Troy, NY
}

\begin{abstract}
Simulated nanoindentation tests on a model metallic glass reveal that the crack initiates inside a shear band via cavitation. The load-displacement curve was shown to be insensitive to the crack initiation but sensitive to subsequent crack propagation. The critical conditions for crack initiation were identified at both the macroscopic and microscopic levels. At the macroscopic level, the indenter geometry affects the overall critical load for crack initiation. Interestingly, the indentation volume at crack initiation appears to be a constant for different indenter geometries, based on which an analytical formula of the critical load as a function of the indenter geometry was derived. At the microscopic level, cavitation occurs once the normal stress perpendicular to the shear band exceeds a temperature-dependent critical cavitation stress. This critical cavitation stress was shown to reduce significantly upon shear deformation.
\end{abstract}

Keywords: nanoindentation, metallic glass, crack initiation, cavitation, molecular simulation 


\section{Introduction}

Metallic glasses are promising structural materials with high strength, high hardness, high wear resistance, reasonable fracture toughness [1-3]. Although at low temperature they often suffer from catastrophic failure [2] in tension tests because of highly localized plastic deformation within shear band, substantial plastic deformation can be introduced into metallic glass under non-uniform stress fields such as indentation, providing an opportunity to study the shear band evolution and its effects on other properties such as fracture and fatigue [4]. Shear band formation under indentation has been extensively studied in both experiments and simulations [4-12]. However, there are few studies on the crack initiation within metallic glasses, partly due to the fact that metallic glasses are not transparent. Thus it is impossible to observe the cracking process using optical methods, such as those used for oxide glass [13, 14]. In general, the indentation crack for metallic glasses has been characterized after the mechanical test. Particularly, the crack initiation has not been well understood, as the crack initiation process is complicated by crack propagation and/or artifacts from sample preparation to expose the crack surface $[15,16]$. Without a mechanistic understanding of crack initiation, it is difficult to rationally improve the crack resistance of metallic glasses.

Crack initiation in brittle materials such as oxide glasses and ceramics has attracted significant research efforts [17-24]. To evaluate the crack initiation and crack resistance of brittle materials, indentation has been widely used. In general, four crack systems have been considered for brittle materials under indentation: (1) radial crack; (2) lateral crack; (3) median crack; (4) ring/cone crack. The initiation of different cracks could occur at different indentation stages [25] (e.g. loading, unloading, or relaxation after unloading). The driving stress component of different cracks is also different according to Yoffe's field theory [26]. The mechanics of crack propagation in brittle materials can be understood fairly well using continuum fracture mechanics $[13,27,28]$, yet a molecular-level understanding of crack initiation is still lacking. There are two crack initiation mechanisms for brittle materials proposed in previous studies $[17,18,20]$ : (1) crack initiates from fortuitous subsurface flaws at the elastic-plastic boundary; (2) crack initiates 
from inhomogeneous shear deformation and intersection of limiting flow lines beneath the indenter. However, the applicability of the above mechanism to metallic glasses is still in question. In our recent study [29], we have shown that crack initiation without shear banding is also possible. Unlike oxide/chalcogenide glasses, which are disordered open networks packed with covalent/ionic bonds [30], metallic glasses have densely packed atomic structures interacting with metallic bonds, which can exhibit significant local shear deformation/shear banding even at room temperature $[2,31]$. As can be seen from experiments $[4-6,32]$ and simulations $[10,12]$, shear band deformation is inhomogeneous, and extends from the most stressed location to elastic zone, which makes it difficult to identify the elastic-plastic deformation boundary. In classical indentation analysis, a well-defined hemispherical/semicylindrical elastic-plastic boundary is assumed in the classical expanding cavity model by Johnson [33-35]. Even though Johnson's analysis has been applied to estimate the plastic zone size for metallic glass containing semi-circular type of shear band $[15,32,36]$, it is unknown whether such analysis is applicable for shear deformation mainly comprised of individual, radially distributed shear band in metallic glasses $[5,6,12]$. Recently, it has been found that the cavitation process can be used to account for the fracture of metallic glass [3, 37-41]. For example, cavitation of metallic glass has been studied under uniaxial loading [41], biaxial loading [38] and hydrostatic loading [37]. However, cavitation and crack initiation in indentation of metallic glass has not been investigated at the molecular-level to our best knowledge.

In this study, displacement-controlled nanoindentation molecular dynamics (MD) simulations are carried out to study crack initiation and deformation in a model metallic glass under the plane-strain condition. Rounded-wedge indenters with different apical radii and tip angles are used in the indentation. A model metallic glass interacting with a modified binary Lennard-Jones $(\mathrm{mBLJ})$ potential is used as the indentation sample. Median cracks are found underneath the indenter during loading. The crack initiation load varies with the indenter geometry. It is found that the crack initiation load of different indenter geometry corresponds to a relative constant critical indentation volume, which may in turn imply an effective critical plastic zone under for 
crack initiation. Furthermore, the cavitation stress inside the shear band is significantly lower than the cavitation stress for samples without shear deformation. In other words, shear deformation reduces the critical cavitation stress, thus assists crack initiation in model metallic glasses under nanoindentation.

\section{Simulation Methodology}

\subsection{Interatomic force field and potential tuning}

A model metallic glass is chosen to study crack initiation under indentation in our MD simulations. The same system has been utilized in the study of intrinsic ductility of glassy solids [42], tensile fracture of metallic glasses [41] and low-cycle fatigue of metallic glass nanowires [43]. The alloy is comprised of two equimolar species with different size: $\mathrm{S}$ (the small atoms) and $\mathrm{L}$ (the large atoms). The interatomic potential used here is the modified binary Lennard-Jones $(\mathrm{mBLJ})$ potential with following form [42]:

$$
\phi_{m B L J}(r)=\left\{\begin{array}{cc}
4 \varepsilon_{\alpha \beta}\left(\frac{\sigma_{\alpha \beta}^{12}}{r^{12}}-\frac{\sigma_{\alpha \beta}^{6}}{r^{6}}\right)-\varepsilon_{\text {cutoff }}, r<r_{\alpha \beta}^{s} \\
4 \varepsilon_{\alpha \beta}\left(\frac{\sigma_{\alpha \beta}^{12}}{r^{12}}-\frac{\sigma_{\alpha \beta}^{6}}{r^{6}}\right)-\varepsilon_{\text {cutoff }} \\
+\varepsilon_{B} \varepsilon_{L L} \cdot \sin ^{2}\left(\pi \frac{r_{\alpha \beta}^{c}-r}{r_{\alpha \beta}^{c}-r_{\alpha \beta}^{s}}\right), & r_{\alpha \beta}^{c}>r \geq r_{\alpha \beta}^{s} \\
0 \quad, \quad & r \geq r_{\alpha \beta}^{c}
\end{array}\right.
$$

where $\varepsilon_{\alpha \beta}$ and $\sigma_{\alpha \beta}$ provide the energy and length scales, respectively. A "bump" energy penalty was added upon the original Wahnstrom system [44] between $r_{\alpha \beta}^{S}\left(=1.5 \sigma_{\alpha \beta}\right)$ and the potential cutoff $r_{\alpha \beta}^{c}$. For all species, the bond energy parameters $(\epsilon)$ are all equal: $\varepsilon_{S S}=\varepsilon_{S L}=$ $\varepsilon_{L L}$. The bond length parameter $(\sigma)$ scales relative to the $S L$ length scale: $\sigma_{S S}=\frac{5}{6} \sigma_{L L}, \sigma_{S L}=$ $\frac{11}{12} \sigma_{L L}$. The potential cutoffs are set differently: $r_{L L}^{c}=2.5 \sigma_{L L}, r_{L S}^{c}=2.2917 \sigma_{L L}, r_{S S}^{c}=$ $2.0833 \sigma_{L L}$ following Ref.[44]. 
The masses for the two types of atoms are: $m_{L}=2 m_{0}, m_{S}=2 m_{0}$ in which $m_{0}$ is the mass unit. The reference time scale $t_{0}=\sigma_{L L} \sqrt{m_{0} / \varepsilon_{L L}}$. All physical quantities have been expressed in SI units in previous report [42]: $\sigma_{L L} \approx 0.27 \mathrm{~nm} ; m_{0} \approx 46 \mathrm{amu} ; \varepsilon_{L L} \approx 0.151 \mathrm{eV} ; t_{0} \approx 0.5 p s$.

The energy penalty, or the "bump", in this mBLJ potential is analogous to the Dzugutov potential [45], which was motivated by the Friedel oscillations [46, 47]. The height of the energy penalty $\varepsilon_{B}$ has been used to control the bond covalency and consequently the intrinsic ductility in this model metallic glass [42]: as $\varepsilon_{B}$ increasing from 0.25 to 0.375 , the metallic glass exhibits a brittle to ductile (BTD) transition due to the increase of bond covalency. To investigate crack initiation, we chose the bump height $\varepsilon_{B}$ to be 0.375 . Thus, this model glass is brittle under uniaxial tension, and has low resistance to crack growth. For more ductile MG model glasses, e.g. Cu-Zr EAM potential [48] with a Poisson's ratio of $\sim 0.4$ (significantly over-predicting experimental value of $\sim 0.35$ ), it would require an extremely large simulation system to observe the crack initiation under indentation. One should note that although the shear band observed in our simulation is far smaller than those observed in experiments, e.g., see Ref.[49], the system does exhibit extensive shear banding activities, prior to crack formation.

\subsection{Sample preparation}

Following the previous report [42], the sample preparation starts from a well-equilibrated liquid at $2100 \mathrm{~K}$. The liquid was subsequently quenched isochorically to $\sim 40 \mathrm{~K}$ in $2.5 \mathrm{~ns}$. We chose the initial density for the liquid so that the residual stress of the as-quenched sample is equal to or close to zero. Small slab-shaped samples were prepared with a size of $16.2 \times 16.2 \times 2.7 \mathrm{~nm}^{3}$ and then tiled 40 across by 20 down to create a single slab. The artificial periodicity in the sample due to periodic-tiling will not result in artificial periodicity in its mechanical response, as the stress distribution in nanoindentation is inhomogeneous [9]. In our simulation the typical dimension of a 
slab is $650 \times 335 \times 2.7 \mathrm{~nm}^{3}$. The total number of atoms in the simulation system is about 30 million. The Young's modulus $(E)$ and the Poisson's ratio $(v)$ of the glassy sample have been measured to be $33 \mathrm{GPa}$ and 0.30 respectively. Other structural properties such as radial distribution function and angular distribution functions can be found elsewhere [42].

\subsection{The setup of the nanoindentation system and virtual indenter}

Fig. 1 shows a schematic of the nanoindentation system used here. Periodic boundary condition applies in the Y-direction. The sample thickness (along the Y-direction) is a constant during nanoindentation simulations. This plane-strain condition has been used in many simulations [9-11], which is also valid in experiments for thick samples $[5,6,12]$. During the indentation test, a wedge-shaped indenter was lowered into the glassy slab samples. Five tip semi-angles $(\theta)$ are used in the current study: $15^{\circ}, 30^{\circ}, 45^{\circ}, 60^{\circ}$ and $68^{\circ}$. The tip end of the indenter is rounded to a semi-cylindrical shape with three different radii $\left(R_{I}=13.5 \mathrm{~nm}, 27 \mathrm{~nm}\right.$ and $\left.54 \mathrm{~nm}\right)$. The indenter is described by imposing an external repulsive force. Once the atom is inside the indenter region, a force of $F_{I i}=-K\left(r_{I i}-R_{I}\right)^{2}$ is applied, where $r_{I i}$ is the shortest distance between atom $i$ and the surface of the wedge-shaped indenter with tip radius of $R_{I} . K$ is chosen to be approximately $1.0 \times 10^{12} \mathrm{~Pa}$. The direction of the force is along the normal direction of the indenter perimeter. In addition to the repulsive force, a frictional force is also imposed with $f_{I i}=-\mu \bullet F_{I i}$, where the negative sign means the direction of the frictional force is against the motion of the atom $i$ relative to the indenter in the tangential direction. The friction coefficient $\mu$ is chosen to be a low value of 0.05 . The slab sample is supported by a flat friction-free wall that interacts with the atoms in the sample following a Lennard-Jones potential only along the X-direction $\left(\phi_{L J}=\right.$ $\left.4 \varepsilon\left[\left(\frac{\sigma}{r}\right)^{12}-\left(\frac{\sigma}{r}\right)^{6}\right], r<r_{c}\right)[50]$. The three potential parameters $\varepsilon, \sigma$ and $r_{c}$ are $0.151 \mathrm{eV}, 0.27$ $\mathrm{nm}$ and $0.675 \mathrm{~nm}$, respectively. Since the wall-sample force is only along the X-direction, sample atoms are allowed to move laterally without any constraints from the wall. Prior to indentation, the whole system is relaxed for $0.05 \mathrm{~ns}$. A loading speed of $30 \mathrm{~m} / \mathrm{s}$ was used, which is two orders of magnitude lower than the sound speed of our glassy samples $(2.3 \sim 2.9 \mathrm{~km} / \mathrm{s})$. During the 
indentation simulation, the system was maintained at a constant temperature of $\sim 15 \% \mathrm{~T}_{\mathrm{g}}$ (the glass transition temperature) or $\sim 40 \mathrm{~K}$, using a Nose-Hoover thermostat [51, 52]. We use LAMMPS package [53] to conduct the nanoindentation MD simulations.

\subsection{Determination of crack initiation load}

In experiments, the threshold load $\left(L_{c}\right)$ for crack initiation of brittle materials can be measured through acoustic emission [22, 54], microseismic monitoring techniques [54] or direct observation of median/radial crack after indentation [55]. In our simulation, two methods with atomic-level accuracy based upon atomic dilatation $[1,56,57]$ and local density change [58] have been used here to directly identify the event of crack initiation and the corresponding loading threshold $\left(L_{c}\right)$. In our density analysis, the indentation samples were divided into small through-thickness volume elements $\left(1.35 \times 2.7 \times 1.35 \mathrm{~nm}^{3}\right)$. Once the local density of a grid in the deformation area below the indenter is lower than the average density of the bulk material by $\sim 15 \%$, the corresponding indentation load is recorded as the $L_{c}$. The cutoff value $15 \%$ is chosen according to the atomic dilation results during crack initiation as shown in Section 3.4. We calculate atomic dilation at the region where crack initiates based on the algorithm by Falk and Langer [59].

\subsection{Determination of critical local thermomechanical state for cavitation}

In order to obtain the critical local thermomechanical state for cavitation, we also conducted two additional mechanical tests (see the insets of Fig. 11): (1) uniaxial tension; (2) biaxial tension. In both cases, smaller samples $\left(7 \times 7 \times 7 \mathrm{~nm}^{3}\right)$ are used, on which plane-strain condition is enforced in the direction (Y-direction) perpendicular with the loading direction (Z-direction; X/Z-direction on $\mathrm{Z} / \mathrm{X}$-plane, respectively). The cavitation stress is defined as the maximum stress that the system can sustain under the above two loading conditions. In general, hydrostatic stress is used to account for cavitation in metallic glass when the loading condition is either biaxial $[38,60]$ or hydrostatic [37]. For the cavitation in a running shear band in uniaxial tension [41], the stress 
component normal to the shear band direction is used. In a well-developed shear band, the normal stress component is equal to the hydrostatic stress [61]. We will compare the above two loading conditions to examine whether the hydrostatic stress or the normal stress dictates cavitation. The two loading conditions can help us understand the controlling stress component under plane strain condition. We do not rule out the possibility of using other stress component (or combinations) to characterize the cavitation under more general loading conditions. To examine the effect of shear deformation on cavitation, the cavitation stress of samples with $40 \%$ shear strain prior to the tension test is also measured. Shear strain of $40 \%$ is used since we found that the cavitation stress stays relative constant with further shear. Cavitation stress at three temperatures $(28 \mathrm{~K}, 70 \mathrm{~K}$ and $140 \mathrm{~K})$ were measured and two strain rates $(0.02 / \mathrm{ns}$ and $0.2 / \mathrm{ns})$ were used for both loading conditions. They are both close to the indentation strain rate $(\sim 0.5 / \mathrm{ns})$ estimated from the ratio between the indentation speed and the indentation depth [62].

\section{Results and Discussion}

\subsection{The indentation process}

Fig. 2 shows the evolution of local shear strain, hydrostatic stress, maximum shear stress and local temperature distribution during the indentation of the model metallic glass sample. It can be seen that a deformation region undergoing severe plastic deformation appears beneath the indenter immediately when the indenter $\left(\theta=30^{\circ}\right.$ and $\left.R_{I}=13.5 \mathrm{~nm}\right)$ penetrates $13.4 \mathrm{~nm}$, or $\sim 4 \%$ of the sample thickness. At temperatures well below the glass transition temperature, metallic glasses usually deform via localized plastic deformation, i.e., shear banding [12]. When the penetration reaches $30.2 \mathrm{~nm}$, several shear bands form below the plastic deformation area. At a penetration of $33.6 \mathrm{~nm}$, the shear band pattern resembles those reported in experimental indentation tests of metallic glasses and indentation simulation using similar indenter geometry $[5,6,12]$. Remarkable pile-ups are also observed when the "wedge part" of the indenter comes to contact with the samples and push the materials sideward. This is common for pyramidal 
indentations in various materials [63-65]. In our simulations, pile-up is suppressed when indenters of either large tip radii or large tip angles are used, consistent with experimental results that use shallow penetration or blunter indenter $[5,6,12]$.

As the indentation penetration increases, a crack initiates inside one of the shear band (as denoted by the arrow at an indentation depth of $33.6 \mathrm{~nm}$ in Fig.2), and propagates downward. The crack can be classified as a 'median crack' with its three distinct features: (1) The crack surface is parallel to the loading direction; (2) The crack forms at a position close to the bottom of the plastic zoom; (3) The crack forms during the loading cycle. Cheng et al. [66] pointed out that, depending the position of the shear-induced flaw, both median cracking and lateral cracking can be initiated. Although lateral crack has not been observed in our simulations, it does not preclude the possibility of lateral cracking (e.g. for a larger simulation system or with a scratching motion). One should note that due to the nature of plane-strain indentation setup, the median crack as shown in Fig.2 will not become a half-penny crack. Radial crack is also absent in the current indentation simulation due to the thin-slab geometry of the simulation system.

The region with maximum hydrostatic tensile stress (indicated by the black arrow in the second row of Fig.2) locates underneath the indentation-induced deformation region, which is close to the elastic/plastic boundary as shown later in Section 3.3 and 3.4. On the other hand, the distribution of maximum shear stress at $13.4 \mathrm{~nm}$ of indentation depth forms a boundary (arrowed in Fig.2) enclosing the indenter. When crack forms around an indentation depth of $33.6 \mathrm{~nm}$, such boundary is broken at the bottom of the deformation region. The local temperature distribution shows that there is a temperature rise at the deformation region undergoing severe plastic deformation (arrowed in Fig.2). The temperature effect on crack initiation are will be discussed in Section 3.4.

\subsection{The load-displacement curve}

Fig. 3 shows the indentation load at different indenter displacements for the sample as shown in Fig. 2. As can be seen from the plot, there is a slope change around $7 \mathrm{~nm}$. This is because the 
indenter is comprised of two parts along its perimeter: the cylindrical apex and the wedge. At the beginning of the indentation loading, only the cylindrical part of the indenter is in contact with the sample. Beyond a certain penetration $\left(h_{c}\right)$, the wedge part of the indenter starts to make contact with the sample, which is illustrated in the inset of Fig. 3. For an indenter of $\theta=30^{\circ}$ and $R_{I}=13.5 \mathrm{~nm}, h_{c}$ is calculated to be $6.75 \mathrm{~nm}$ ignoring pile-ups. For the crack initiation which is around $32 \mathrm{~nm}$ of displacement (shown by the dot in Figure 3), there is no discernable change in the load-displacement curve. Upon further crack propagation, the load drops significantly after a displacement of about $40 \mathrm{~nm}$ (see black arrow). It appears that the load-displacement curve is not sensitive to crack initiation, but is sensitive to crack propagation.

\subsection{Macroscopic crack initiation condition: the critical crack initiation load}

In order to obtain the relation between the indenter geometry and the crack initiation load, indentation simulations on this model metallic glass sample are conducted using a series of indenter semi-angles $(\theta)$ and apical radii $\left(R_{I}\right)$. Fig. 4 shows the crack initiation load $\left(L_{c}\right)$ obtained from the local density measurement (as detailed in Section 2.4) for indentation using different indenter geometries. For the indenter with $R_{I}=13.5 \mathrm{~nm}, L_{c}$ increases monotonically with $\theta$. However, for the indenter with $R_{I}=54 \mathrm{~nm}, L_{c}$ is relatively constant for $\theta$ ranging from $0^{\circ}$ up to $45^{\circ}$. At large $\theta$, all the curves seem to converge and increase rapidly with $\theta$. This convergence indicates that $L_{c}$ is not sensitive to the radius of the indenter tip for blunt indenters. The observed trend of the crack initiation load as a function of indenter semi-angle for large indenter angle is similar to the experimental results on oxide glasses [55]. Here, a relation between the indentation crack initiation threshold $\left(L_{c}\right)$ and the indenter geometry $\left(\theta\right.$ and $\left.R_{I}\right)$ can be established through the indentation model by Chiang et al. [23, 24, 67] under plane-strain condition. The essence of Chiang's model is that the plastic zone volume is determined by plastic work of indentation, which can be calculated from the indentation volume and the indentation pressure. The following assumptions/arguments are made based on Chiang's model:

(i) It is assumed that the crack initiates at the bottom of the elastic/plastic boundary, which is also an assumption of the crack initiation mechanisms proposed by others $[17,18,66]$. Therefore, we 
focus on the bottom area of the plastic zone in the following analysis. This is consistent with our simulation results that the crack initiation always occurs near the bottom of the plastic zone.

(ii) The plastic zone volume $V_{p}$ (the volume of plastically deformed region underneath the indenter) is determined by the plastic work of indentation $W_{p}$, such that $V_{p}=C * W_{p}$, where $C$ is a proportionality constant. Since $W_{p}$ is the product of average indentation pressure $p$, defined in (iii), and indentation volume $\Delta V$ (the volume of the indent), $V_{p}$ can be written as a function of $p$ and $\Delta V$. According to Fig.1, from the geometry of the rounded wedge indenter we have:

$$
\begin{aligned}
& V_{p}=C * p * \Delta V \\
& =\left\{\begin{array}{c}
C * p * l * R_{I}^{2}\left\{\left[\sin ^{-1}\left(\frac{r}{R_{I}}\right)-\frac{r}{R_{I}} * \sqrt{\left.\left.1-\left(\frac{r}{R_{I}}\right)^{2}\right]\right\},} \quad r<R_{I} * \cos \theta\right.\right. \\
C * p * l *\left\{\frac{R_{I}^{2}}{2}[\pi-2 \theta-\sin (2 \theta)]+\left(r^{2}-\left(R_{I} * \cos \theta\right)^{2}\right) * \cot \theta\right\} \\
, \quad r \geq R_{I} * \cos \theta
\end{array}\right.
\end{aligned}
$$

Where $l$ is the sample thickness and $r$ is half of the width of the contact area between the indenter and the sample (see inset of Fig.3). The first condition $\left(r<R_{I}^{*} \cos \theta\right)$ corresponds to the situation that only the spherical part of the indenter (see the shaded area in the inset of Fig.3) is in contact with the materials, for which the $\Delta V$ is not a function of $\theta$. The second condition $\left(r \geq R_{I}^{*} \cos \theta\right)$ corresponds to the situation when the wedge part also makes contact with the materials.

(iii) Indentation pressure $p$ (or hardness $H$ ) can be defined as,

$$
p=\frac{L}{2 r}
$$

where $L$ is the load in the unit of $\mathrm{nN} / \mathrm{nm}$. In our indentation simulation with different indenter geometries $\left(\theta\right.$ and $\left.R_{I}\right)$ and with two parallel samples, $p$ is found to be $1.5 \pm 0.4 \mathrm{GPa}$.

(iv) The stress distribution before the crack initiation is dictated by the indentation volume $(\Delta V)$. During the indentation process, the plastic zone grows and the local stress distribution varies 
accordingly. Fig.5A shows the distribution of the stress $\left(\sigma_{z z}\right)$ component pertinent to median crack along the X-direction (as shown in the inset of Fig.5A) at different indentation depths for an indenter of $\theta=15^{\circ}$ and $R_{I}=27 \mathrm{~nm}$. Distances along X-and Z- are normalized by the radius $a$ of the indentation core (inset of $(\mathrm{A}))$ whose volume equals the indentation area $\Delta S\left(\pi a^{2}=4 \bullet \Delta S\right)$. The peak tensile stress increases progressively with the indenter penetration and reaches the highest value for an indentation depth of $28.5 \mathrm{~nm}$ at which the crack initiates. Fig.5B shows the $\sigma_{z z}$ distribution for different indentation depths along the Z-direction (as shown in the inset of Fig.5A) and at the depth of peak $\sigma_{z z}$ in Fig. 5A. The fact that $\sigma_{z z}$ decreases with $|z|$ and that it reaches maximum value somewhere below the sample surface $(x>0)$ is consistent with the assumption (i) that crack initiation always occurs at the bottom of the plastic zone. It is interesting to see whether the maximum $\sigma_{z z}$ corresponds to any critical indentation volume $\Delta V$ (or indentation area $\Delta S$ because all the samples have the same thickness $l$ ). Fig.5C shows the maximum $\sigma_{z z}$ at different indentation area $\Delta S$ for all the indenter geometries in use. As can be clearly seen, the crack initiation marked by (प) occurs in the elliptical region where $\sigma_{z z} \approx 0.5 \sim 0.6 \mathrm{GPa}$ and $\Delta S \approx 1000 \sim 1500 \mathrm{~nm}^{2}$. Therefore a critical indentation area $\Delta S_{\text {critical }}$ can be defined using the average of $\Delta S$ at which crack initiates. The $\Delta S_{\text {critical }}$ is calculated to be $1300 \pm 203 \mathrm{~nm}^{2}$ from the indentation of two parallel samples using all the indenter geometries listed in Section 2.3. Substitute $\Delta S_{\text {critical }}$ and Eq. (3) into Eq. (2), the following equation can be obtained:

$$
\Delta S_{\text {critical }}=\left\{\begin{array}{cl}
R_{I}^{2}\left(\left[\sin ^{-1}\left(\frac{L_{c}}{2 p * R_{I}}\right)-\frac{L_{c}}{2 p * R_{I}} * \sqrt{\left.1-\left(\frac{L_{c}}{2 p * R_{I}}\right)^{2}\right]},\right.\right. & \frac{L_{c}}{2 p}<R_{I} * \cos \theta \\
\frac{R_{I}^{2}}{2}[\pi-2 \theta-\sin (2 \theta)]+ & \\
\left(\left(\frac{L_{c}}{2 p}\right)^{2}-\left(R_{I} * \cos \theta\right)^{2}\right) * \cot \theta, & \frac{L_{c}}{2 p} \geq R_{I} * \cos \theta
\end{array}\right.
$$


Where $L_{c}$ is the crack initiation load. For most of the indenters (except for the indenters with $R_{I}=$ $54 \mathrm{~nm}$ and $\theta=15^{\circ}$ or $30^{\circ}$ ) in used in our indentation simulation, the spherical part of the indenter has an area less than $1300 \mathrm{~nm}^{2}$, therefore the second condition is mostly satisfied here. After rearrangement, the critical load $L_{c}$ as an explicit function of both $\theta$ and $R_{I}$ can be obtained under the second condition above:

$L_{c}=2 p \sqrt{\tan \theta\left[\Delta S_{\text {critical }}-R_{I}^{2} *\left(\frac{\pi}{2}-\theta\right)\right]+R_{I}^{2}}$

With $p, \Delta S_{\text {critical }}, R_{I}, \theta$ in Eq. (4) known or measured in our simulation, one can plot $L_{c}$ (crack initiation load) as a function of indenter angle $\theta$ for different indenter radii $R_{I}$ as shown in Fig.4. It can be seen that the $L_{c}\left(\theta, R_{I}\right)$ is in excellent agreement with the measured $L_{c}$ from all the indenter geometries in used. We note here that although there is no direct correlation between the indentation size $\Delta S$ and the stress $\sigma_{z z}$, the use of the critical indentation area $\Delta S_{\text {critical }}$ provides a convenient way to establish the relation (Eq.(4) and Eq.(5)) between the critical indentation load $L_{c}$ and the indenter geometry $\left(\theta\right.$ and $\left.R_{I}\right)$ in use in our simulation. It is not clear if the $\Delta S_{\text {critical }}$ would be applicable for indenter geometries significantly deviating from those investigated here.

\subsection{Microscopic crack initiation condition: the critical local thermomechanical state for cavitation}

The macroscopic crack initiation criterion in the above section is based on a critical indentation volume, which corresponds to a critical local stress distribution underneath the indenter. Under such stress distribution, certain region of the material exhibits cavitation. In this section, we will focus on identifying the specific local thermomechanical state for cavitation.

Fig.6A is a close-up view of the indentation region (see Fig. 2, local shear distribution when the penetration depth equals $33.6 \mathrm{~nm}$ ) at the crack initiation. The bottom of the plastic zone corresponding to the maximum $\sigma_{z z}$ is shown in Fig.6A by the dashed curve line. A further close-up view of the cavitation region in the dashed box in Fig.6A is shown in Fig.6B in which 
small cavities form within the shear band and coalesce to become the tip of the crack. These results are the first simulation to show that cavitation process accounts for crack initiation in the indentation of the metallic glass. Fig. $6 \mathrm{C}$ plots the local shear strain and atomic dilation $[1,59]$ evolution during the indentation at the circular region in Fig. 6B. Before the crack initiates (about $1.1 \mathrm{~ns}$ ), the local shear strain reaches $\sim 15 \%$ and yet the dilation of the system is less than $4 \%$. The dilation increases up to more than $20 \%$ once cavitation starts. In the literature, dilation as high as $15 \%$ has been shown to lead to fracture through cavitation $[1,38]$. Since the atomic dilation is very sensitive to cavitation, it can be used to identify the event of crack initiation (see Section 2.4).

Fig.6D shows the thermomechanical condition evolution at the cavitation position as denoted in Fig.6B. The normal stress components perpendicular to $\left(\sigma_{z^{\prime} z^{\prime}}\right)$, parallel with $\left(\sigma_{x^{\prime} x}\right)$ the shear band, the shear stress $\left(\sigma_{x^{\prime}} z^{\prime}\right)$, and the local temperature along the shear band were extracted. The data points in Fig.6D are average values from the circular region (Fig. $6 \mathrm{~B}, \sim 2.7 \mathrm{~nm}$ in radius) at the cavitation location through the thickness of the sample. As can be seen, the stress along the shear band increases gradually during indentation. Once the two stress $\sigma_{x^{\prime} x^{\prime}}$ and $\sigma_{z^{\prime} z^{\prime}}$ reach maximum, the cavitation occurs and the stresses drop down to zero dramatically. Prior to cavitation, the local temperature stays at the initial temperature $(\sim 40 \mathrm{~K})$. However, it increases rapidly upon cavitation due to significant local plastic deformation from shear banding and bond breaking. Under the same scheme, Fig.7 shows the thermomechanical state of the cavitation position of indentation simulations using two indenter semi-angles $\left(30^{\circ}\right.$ and $\left.60^{\circ}\right)$ and three indenter radii $(13.5,27$ and $54 \mathrm{~nm}$ ). The arrows are eye guides showing the thermomechanical state evolution direction. It is clear that the thermomechanical conditions must reach some critical state before the cavitation occurs. Such critical state is determined by both the local stress and the local temperature. For the $30^{\circ}$ indenter semi-angle case, the local temperature does not increase much before crack initiation (also shown in Fig. 6D), therefore, the local stress state seems to be the dominant factor for crack initiation in this situation. However, temperature increases become more significant for the $60^{\circ}$ tip semi-angle case. This is probably because blunter indenter tends to cause more plastic 
deformation, which heats the sample before cavitation occurs. Sharp indenter, instead, tends to cut the sample and promote cavitation with less plastic deformation.

In order to understand the critical thermomechanical state for crack initiation, we measured the cavitation stress under two homogeneous loading conditions (See Section 3.2): (1) uniaxial tension; (2) biaxial tension. In the uniaxial tension condition as shown in the inset of Fig.8A, the reported stress component is $\sigma_{z z}$ which is along the loading direction; in the case of biaxial tension as shown in the inset of Fig.8B, both $\sigma_{x x}$ and $\sigma_{z z}$ were measured and are close to each other throughout the loading process, therefore only $\sigma_{z z}$ is reported. For both situations, in order to study the shear effect on the cavitation stress, we conducted additional simulations where a shear band is introduced prior the uniaxial or biaxial tensile loading (termed pre-sheared samples). Cavitation stresses with or without pre-shear are both presented in Fig.8.

\subsection{Understanding the local thermomechanical condition for cavitation}

Several observations can be made based on simulation results from Fig. 8 and previous Sections.

First, the cavitation stresses using the same indentation strain rate are quite close to each other for both uniaxial tension and biaxial tension conditions. For example, at $28 \mathrm{~K}$ and without pre-shear, the cavitation stress is about $0.85 \mathrm{GPa}$ for a strain rate of $0.2 / \mathrm{ns}$. Therefore, for our model metallic glass, we can use the stress component $\left(\sigma_{z^{\prime} z^{\prime}}\right.$, in Fig.8B) perpendicular with the shear band that dictates the cavitation process. Note, in well-developed running shear band, the local $\sigma_{z^{\prime}} z^{\prime}$ has been observed to be equal to the local hydrostatic stress in the shear band [61], which results from the atomic disorder induced liquid feature of the running shear band. Here under nanoindentation, the shear band has not been fully developed when cavitation occurs, therefore $\sigma_{z^{\prime} z^{\prime}}$ does not equal the local hydrostatic stress [61]. Second, as indicated by the arrows in Fig.8, the cavitation stress threshold is reduced by almost 50\% when the pre-shear (or shear band) is introduced. Therefore, cavitation becomes much easier inside a shear band than in the undeformed area. This explains well the observation in both Fig. 2 and Fig.6 that the crack initiation through cavitation occurs within the shear band. Note, our observation that shear intrinsically weakens the material is different from a previous study that attributes the preferable shear band cavitation to the plastic 
confinement induced hydrostatic stress elevation inside shear band [60]. In more ductile model glasses, shear flow rather than shear deformed state is required to reduce the cavitation threshold to a significant level [41]. Third, the cavitation stress decreases with temperature and increases with normal loading strain rate, which is consistent with the previous study [41].

To better understand the critical thermomechanical state of cavitation, we could apply the cavitation line (e.g. the one corresponding to a strain rate of $0.02 / \mathrm{ns}$ ) obtained in Fig. 8 to the local material under indenter in Fig.7. It is evident that when the local thermomechanical state reaches the cavitation line, cavitation occurs. For example, when an indenter $\left(\theta=30^{\circ}\right.$ and $\left.R_{I}=27 \mathrm{~nm}\right)$ is used, crack will initiate once the local stress $\left(\sigma_{z z}\right)$ reaches $0.4 \mathrm{GPa}$ at $40 \mathrm{~K}$ according to the cavitation line.

Lastly, we will discuss how one can comprehend crack initiation at both macroscopic level and microscopic level. With the indenter penetrating into the model glass sample, a plastic region forms and grows beneath the indenter. Note, this plastic region represents the mechanical agitation of indentation, without the need of considering the indenter geometry, which determines the subsequent local thermomechanical distribution of the tested sample. This view links the macroscopic cavitation criterion to the microscopic cavitation criterion. As indentation proceeds, the plastic region volume increases, causing the increase of temperature and local shear stress. Thus, the local thermomechanical state evolves from the low temperature-low stress state to high temperature-high stress states. Once the thermomechanical state reaches the cavitation line, cavitation occurs.

\section{Conclusion}

The crack initiation in indentation of a model metallic glass is studied by molecular dynamics simulations. The load-displacement curve is shown to be insensitive to the crack initiation but sensitive to subsequent crack propagation. Macroscopically, the crack initiation load for different indenter shapes are obtained with the assumption of a critical indentation volume. At the microscopic level, a critical local thermomechanical states underneath the indenter for cavitation 
were identified. From uniaxial and biaxial tension tests, the cavitation stress is shown to be significantly reduced by the shear band deformation.

\section{Acknowledgements}

Y. J. Y. is grateful for the intern support of Corning Incorporated and many stimulating discussions with Peter Lezzi, Sushmit Goyal, Vijay Subramanian, and Adama Tandia from Corning Incorporated. Y. F. S. would like to thank the support from the National Science Foundation under grant DMR-1207439, and the Gordon S. Fulcher sabbatical program at Corning Incorporated. L. P. H. acknowledges the financial support from the National Science Foundation under grant No. DMR-1255378, and the Gordon S. Fulcher sabbatical program at Corning Incorporated. Molecular dynamics simulations were carried out in LAMMPS using supercomputers in the Computational Center for Innovations (CCI) at RPI. 


\section{Figure Captions}

Fig.1 Illustration of the nanoindentation simulation geometry.

Fig.2 Distribution of local shear strain, hydrostatic stress, maximum shear stress and local temperature at different indenter penetrations. The indenter radius $\left(R_{I}\right)$ is $13.5 \mathrm{~nm}$ and the indenter semi-angle $(\theta)$ is $30^{\circ}$.

Fig.3 Load versus displacement during loading and unloading phases of indentation. The maximum indenter penetration for all indentation simulations is $\sim 20 \%$ of the sample thickness ( $\sim 300 \mathrm{~nm})$. The black arrow (A) denotes the moment when the crack in the sample starts propagating. The red arrow (B) denotes when the wedge part of the indenter makes contact with the sample. The blue dot $(\mathrm{C})$ is the crack initiation point. $h$ and $r$ in the inset denote the indenter penetration and half of the contact width. $h_{c}$ denotes the critical penetration beyond which the wedge part makes contact with the sample.

Fig.4 Critical load versus indenter tip angle. The data are extracted from two independent samples.

Fig.5 (A) $\sigma_{z z}$ distribution along centerline $\mathrm{x}$ of the sample (A) and $\mathrm{z}$ direction (B) at different indentation depths (see the legend). Both directions are illustrated in the inset of (A) and the curves in (B) are plotted along the $\mathrm{x}$ coordinate corresponding to the peaks in (A). The results are from indentation using an indenter of $\theta=15^{\circ}$ and $R_{I}=27 \mathrm{~nm}$. Distances along $\mathrm{x}$ and $\mathrm{z}$ are normalized by the radius $a$ of the indentation core (inset of (A)) whose volume equals the indentation area $\Delta \mathrm{S}$. (C) variation of the maximum $\sigma_{z z}$ with indentation area $\Delta S\left(\Delta S=0.25 \pi a^{2}\right)$. The indenter parameters $\left(2 \theta\right.$ and $\left.R_{I}\right)$ are given in the legend and the crack initiation for each indentation simulation is marked by $(\square)$. Data from two parallel samples are included.

Fig.6 Snapshots (A) and (B) from indentation MD simulation at $1.2 \mathrm{~ns}$, showing shear bands and cavity nucleation within shear bands. The legend specifies the local shear strain. (C) local shear strain and atomic dilation evolution in the circled cavity region as shown in (B). (D) Stress and temperature evolution at the cavity region as indicated by the arrow in (B). 
Fig.7 Cavitation map in the normal stress $\left(\sigma_{z z}\right)$ and temperature (T) domain with the thermomechanical state evolution of the cavitation sites during indentation tests. Results from two indenter tip semi-angles $(\theta)$ (left: $30^{\circ}$ and right: $60^{\circ}$ ) and three tip radius $\left(R_{I}\right)$ are included. The cavitation line is measured in Fig. 8.

Fig. 8 Thermo-mechanical conditions for cavitation of small samples $\left(\varepsilon_{B}=0.375\right)$ in the stress and temperature domain. Both uniaxial tension (A) and biaxial tension (B) conditions were used. $\dot{\varepsilon}$ represents either the uniaxial tension strain rate or the biaxial tension strain rate. In both tests, two pre-shears $(0 \%$ and $40 \%)$ were applied to the samples. As can be seen from above, the cavitation stresses from both conditions are quite close to each other. 


\section{References}

[1] Y.-J. Wang, M. Jiang, Z. Tian, L. Dai. Direct atomic-scale evidence for shear-dilatation correlation in metallic glasses, Scripta Materialia (2015).

[2] C.A. Schuh, T.C. Hufnagel, U. Ramamurty. Mechanical behavior of amorphous alloys, Acta Materialia 55 (2007) 4067-4109.

[3] M.D. Demetriou, M.E. Launey, G. Garrett, J.P. Schramm, D.C. Hofmann, W.L. Johnson, R.O. Ritchie. A damage-tolerant glass, Nature materials 10 (2011) 123-128.

[4] C. Packard, C. Schuh. Initiation of shear bands near a stress concentration in metallic glass, Acta Materialia 55 (2007) 5348-5358.

[5] A. Antoniou, A. Bastawros, B. Biner. Experimental observations of deformation behavior of bulk metallic glasses during wedge-like cylindrical indentation, Journal of materials research 22 (2007) 514-524.

[6] A. Antoniou, A. Bastawros, C. Lo, S. Biner. Deformation behavior of a zirconium based metallic glass during cylindrical indentation: in situ observations, Materials Science and Engineering: A 394 (2005) 96-102.

[7] P. Donovan. Plastic flow and fracture of Pd40Ni40P20 metallic glass under an indentor, Journal of materials science 24 (1989) 523-535.

[8] L. Gu, L. Xu, Q. Zhang, D. Pan, N. Chen, D.V. Louzguine-Luzgin, K.-F. Yao, W. Wang, Y. Ikuhara. Direct in situ observation of metallic glass deformation by real-time nano-scale indentation, Scientific reports 5 (2015).

[9] Y. Shi, M.L. Falk. Structural transformation and localization during simulated nanoindentation of a noncrystalline metal film, Applied Physics Letters 86 (2005) 011914. [10] Y. Shi, M.L. Falk. Stress-induced structural transformation and shear banding during simulated nanoindentation of a metallic glass, Acta materialia 55 (2007) 4317-4324.

[11] Y. Shi, M.L. Falk. Simulations of nanoindentation in a thin amorphous metal film, Thin Solid Films 515 (2007) 3179-3182.

[12] C. Su, L. Anand. Plane strain indentation of a Zr-based metallic glass: Experiments and numerical simulation, Acta materialia 54 (2006) 179-189.

[13] B. Lawn, M. Swain. Microfracture beneath point indentations in brittle solids, Journal of materials science 10 (1975) 113-122.

[14] T. Rouxel. Driving force for indentation cracking in glass: composition, pressure and temperature dependence, Philosophical Transactions of the Royal Society of London A: Mathematical, Physical and Engineering Sciences 373 (2015) 20140140.

[15] U. Ramamurty, S. Jana, Y. Kawamura, K. Chattopadhyay. Hardness and plastic deformation in a bulk metallic glass, Acta Materialia 53 (2005) 705-717.

[16] V. Keryvin, X. Vu, V. Hoang, J. Shen. On the deformation morphology of bulk metallic glasses underneath a Vickers indentation, Journal of Alloys and Compounds 504 (2010) S41-S44. [17] B. Lawn, A. Evans. A model for crack initiation in elastic/plastic indentation fields, Journal of Materials Science 12 (1977) 2195-2199. 
[18] J. Hagan. Micromechanics of crack nucleation during indentations, Journal of Materials Science 14 (1979) 2975-2980.

[19] J. Hagan. Shear deformation under pyramidal indentations in soda-lime glass, Journal of Materials Science 15 (1980) 1417-1424.

[20] J. Hagan, M.V. Swain. The origin of median and lateral cracks around plastic indents in brittle materials, Journal of Physics D: Applied Physics 11 (1978) 2091.

[21] M. Swain, J. Hagan. Indentation plasticity and the ensuing fracture of glass, Journal of Physics D: Applied Physics 9 (1976) 2201.

[22] J. Lankford, D.L. Davidson. The crack-initiation threshold in ceramic materials subject to elastic/plastic indentation, Journal of Materials Science 14 (1979) 1662-1668.

[23] S. Chiang, D. Marshall, A. Evans. The response of solids to elastic/plastic indentation. I. Stresses and residual stresses, Journal of Applied Physics 53 (1982) 298-311.

[24] S. Chiang, D. Marshall, A. Evans. The response of solids to elastic/plastic indentation. II. Fracture initiation, Journal of Applied Physics 53 (1982) 312-317.

[25] R.F. Cook, G.M. Pharr. Direct observation and analysis of indentation cracking in glasses and ceramics, Journal of the American Ceramic Society 73 (1990) 787-817.

[26] E. Yoffe. Elastic stress fields caused by indenting brittle materials, Philosophical Magazine A 46 (1982) 617-628.

[27] B.R. Lawn, E. Fuller. Equilibrium penny-like cracks in indentation fracture, Journal of Materials Science 10 (1975) 2016-2024.

[28] H. Exner. The influence of sample preparation on Palmqvists method for toughness testing of cemented carbides, Trans Met Soc AIME 245 (1969) 677-683.

[29] J. Luo, K.D. Vargheese, A. Tandia, G. Hu, J.C. Mauro. Crack nucleation criterion and its application to impact indentation in glasses, Scientific Reports 6 (2016) 23720.

[30] J.C. Mauro, A.K. Varshneya. Modeling of rigidity percolation and incipient plasticity in germanium-selenium glasses, Journal of the American Ceramic Society 90 (2007) 192-198.

[31] A. Greer, Y. Cheng, E. Ma. Shear bands in metallic glasses, Materials Science and Engineering: R: Reports 74 (2013) 71-132.

[32] S. Jana, R. Bhowmick, Y. Kawamura, K. Chattopadhyay, U. Ramamurty. Deformation morphology underneath the Vickers indent in a Zr-based bulk metallic glass, Intermetallics 12 (2004) 1097-1102.

[33] K.L. Johnson, K.L. Johnson. Contact mechanics, Cambridge university press, 1987.

[34] K. Johnson. The correlation of indentation experiments, Journal of the Mechanics and Physics of Solids 18 (1970) 115-126.

[35] D. Kramer, H. Huang, M. Kriese, J. Robach, J. Nelson, A. Wright, D. Bahr, W. Gerberich. Yield strength predictions from the plastic zone around nanocontacts, Acta Materialia 47 (1998) 333-343.

[36] S. Jana, U. Ramamurty, K. Chattopadhyay, Y. Kawamura. Subsurface deformation during Vickers indentation of bulk metallic glasses, Materials Science and Engineering: A 375 (2004) 1191-1195. 
[37] P. Guan, S. Lu, M.J.B. Spector, P.K. Valavala, M.L. Falk. Cavitation in Amorphous Solids, Physical Review Letters 110 (2013) 185502.

[38] P. Murali, T.F. Guo, Y.W. Zhang, R. Narasimhan, Y. Li, H.J. Gao. Atomic Scale

Fluctuations Govern Brittle Fracture and Cavitation Behavior in Metallic Glasses, Physical

Review Letters 107 (2011) 215501.

[39] E. Bouchaud, D. Boivin, J.-L. Pouchou, D. Bonamy, B. Poon, G. Ravichandran. Fracture through cavitation in a metallic glass, EPL (Europhysics Letters) 83 (2008) 66006.

[40] R. Maaß, P. Birckigt, C. Borchers, K. Samwer, C. Volkert. Long range stress fields and cavitation along a shear band in a metallic glass: The local origin of fracture, Acta Materialia 98 (2015) 94-102.

[41] J. Luo, Y. Shi. Tensile fracture of metallic glasses via shear band cavitation, Acta Materialia 82 (2015) 483-490.

[42] Y. Shi, J. Luo, F. Yuan, L. Huang. Intrinsic ductility of glassy solids, Journal of Applied Physics 115 (2014) 043528.

[43] J. Luo, K. Dahmen, P.K. Liaw, Y. Shi. Low-cycle fatigue of metallic glass nanowires, Acta Materialia 87 (2015) 225-232.

[44] Y. Shi, M.L. Falk. Atomic-scale simulations of strain localization in three-dimensional model amorphous solids, Physical Review B 73 (2006) 214201.

[45] M. Dzugutov. Glass formation in a simple monatomic liquid with icosahedral inherent local order, Physical Review A 46 (1992) R2984.

[46] M. Dzugutov, K.-E. Larsson, I. Ebbsjö. Pair potential in liquid lead, Physical Review A 38 (1988) 3609.

[47] W.A. Harrison. Pseudopotentials in the Theory of Metals, 1966, 336 P. W. A. BENJAMIN, INC., NEW YORK (1966).

[48] Y.Q. Cheng, A.J. Cao, E. Ma. Correlation between the elastic modulus and the intrinsic plastic behavior of metallic glasses: The roles of atomic configuration and alloy composition, Acta Materialia 57 (2009) 3253-3267.

[49] P. Tandaiya, R. Narasimhan, U. Ramamurty. On the mechanism and the length scales involved in the ductile fracture of a bulk metallic glass, Acta Materialia 61 (2013) 1558-1570. [50] J.E. Jones. On the Determination of Molecular Fields. II. From the Equation of State of a Gas, Proceedings of the Royal Society of London A: Mathematical, Physical and Engineering Sciences 106 (1924) 463-477.

[51] W.G. Hoover. Canonical dynamics: Equilibrium phase-space distributions, Physical Review A 31 (1985) 1695-1697.

[52] S. Nosé. A unified formulation of the constant temperature molecular dynamics methods, The Journal of Chemical Physics 81 (1984) 511-519.

[53] S. Plimpton. Fast parallel algorithms for short-range molecular dynamics, Journal of computational physics 117 (1995) 1-19.

[54] M. Cai, P.K. Kaiser, Y. Tasaka, T. Maejima, H. Morioka, M. Minami. Generalized crack initiation and crack damage stress thresholds of brittle rock masses near underground excavations, International Journal of Rock Mechanics and Mining Sciences 41 (2004) 833-847. 
[55] T. Gross. Deformation and cracking behavior of glasses indented with diamond tips of various sharpness, Journal of Non-Crystalline Solids 358 (2012) 3445-3452.

[56] E. Eberhardt, D. Stead, B. Stimpson, R. Read. Identifying crack initiation and propagation thresholds in brittle rock, Canadian Geotechnical Journal 35 (1998) 222-233.

[57] C. Martin, N. Chandler. The progressive fracture of Lac du Bonnet granite. International Journal of Rock Mechanics and Mining Sciences \& Geomechanics Abstracts, vol. 31: Elsevier, 1994. p.643-659.

[58] V. Schmidt, H. Rösner, M. Peterlechner, G. Wilde, P.M. Voyles. Quantitative Measurement of Density in a Shear Band of Metallic Glass Monitored Along its Propagation Direction, Physical Review Letters 115 (2015) 035501.

[59] M. Falk, J. Langer. Dynamics of viscoplastic deformation in amorphous solids, Physical Review E 57 (1998) 7192.

[60] P. Murali, R. Narasimhan, T. Guo, Y. Zhang, H. Gao. Shear bands mediate cavitation in brittle metallic glasses, Scripta Materialia 68 (2013) 567-570.

[61] J. Luo, Y. Shi. The local stress state of a running shear band in amorphous solids, Journal of Materials Research (2015) 1-9.

[62] R.J. Anton, G. Subhash. Dynamic Vickers indentation of brittle materials, Wear 239 (2000) 27-35.

[63] C. Tang, Y. Li, K. Zeng. Characterization of mechanical properties of a Zr-based metallic glass by indentation techniques, Materials Science and Engineering: A 384 (2004) 215-223.

[64] T. Mulhearn. The deformation of metals by Vickers-type pyramidal indenters, Journal of the Mechanics and Physics of Solids 7 (1959) 85-88.

[65] K. Peter. Densification and flow phenomena of glass in indentation experiments, Journal of Non-Crystalline Solids 5 (1970) 103-115.

[66] W. Cheng, I. Finnie. A mechanism for sub-surface median crack initiation in glass during indenting and scribing, Journal of materials science 25 (1990) 575-579.

[67] B.R. Lawn, A. Evans, D. Marshall. Elastic/plastic indentation damage in ceramics: the median/radial crack system, Journal of the American Ceramic Society 63 (1980) 574-581. 


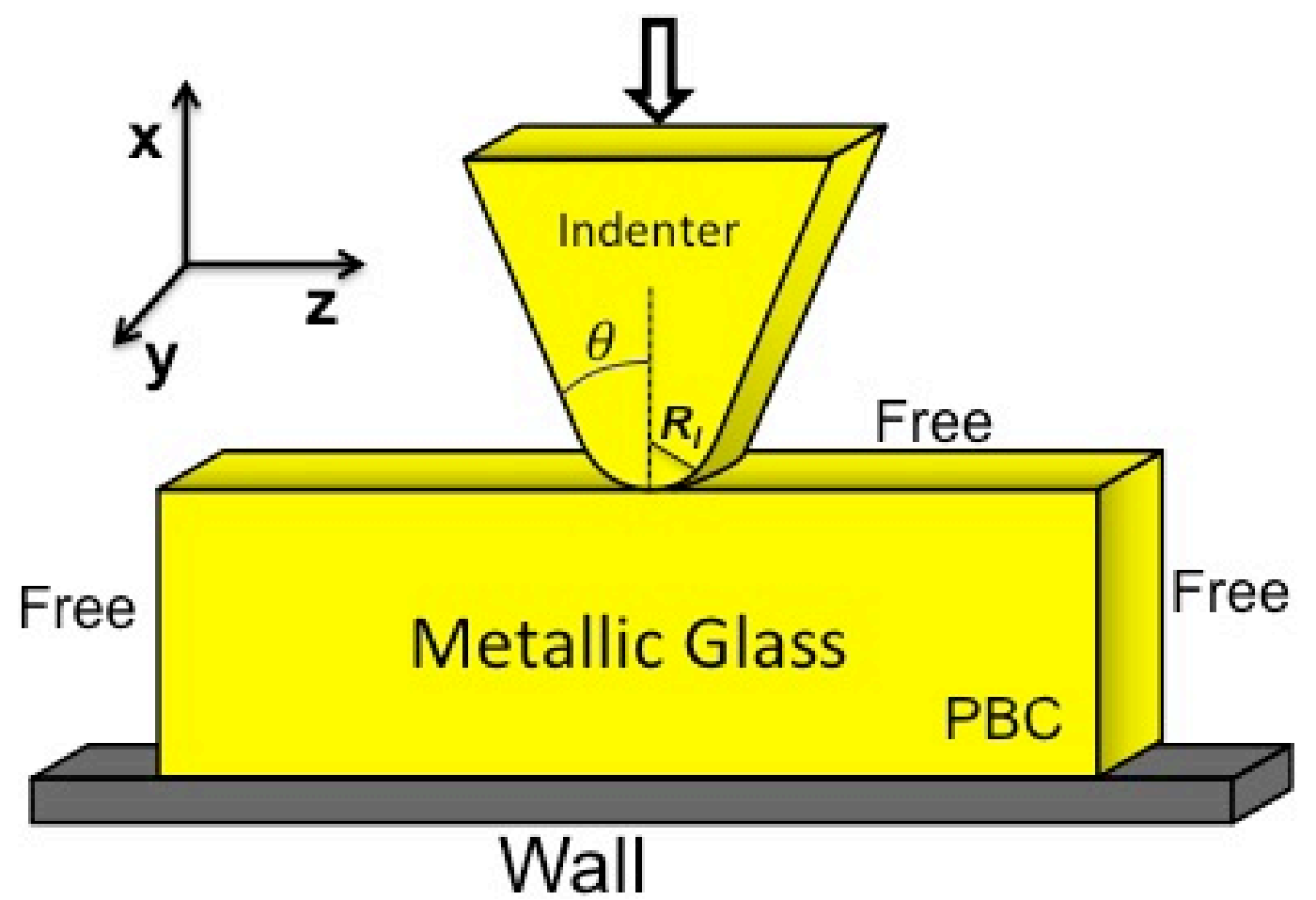

Fig.1 Illustration of the nanoindentation simulation geometry. 
Local shear

Hydrostatic stress

Maximum shear stress

\section{$13.4 \mathrm{~nm}$}

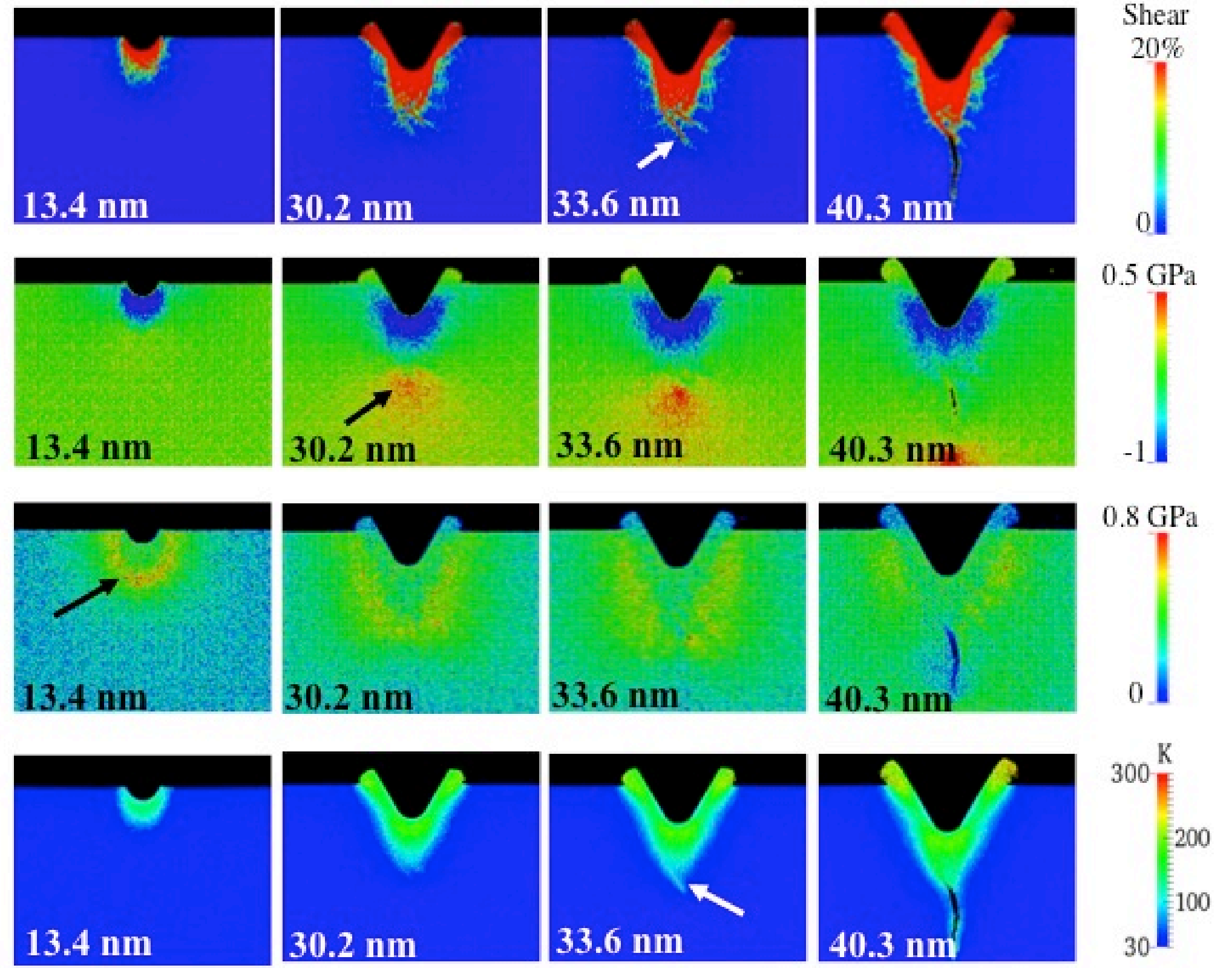

Shear

Fig.2 Distribution of local shear strain, hydrostatic stress, maximum shear stress and local temperature at different indenter penetrations. The indenter radius $\left(R_{I}\right)$ is $13.5 \mathrm{~nm}$ and the indenter semi-angle $(\theta)$ is $30^{\circ}$. 


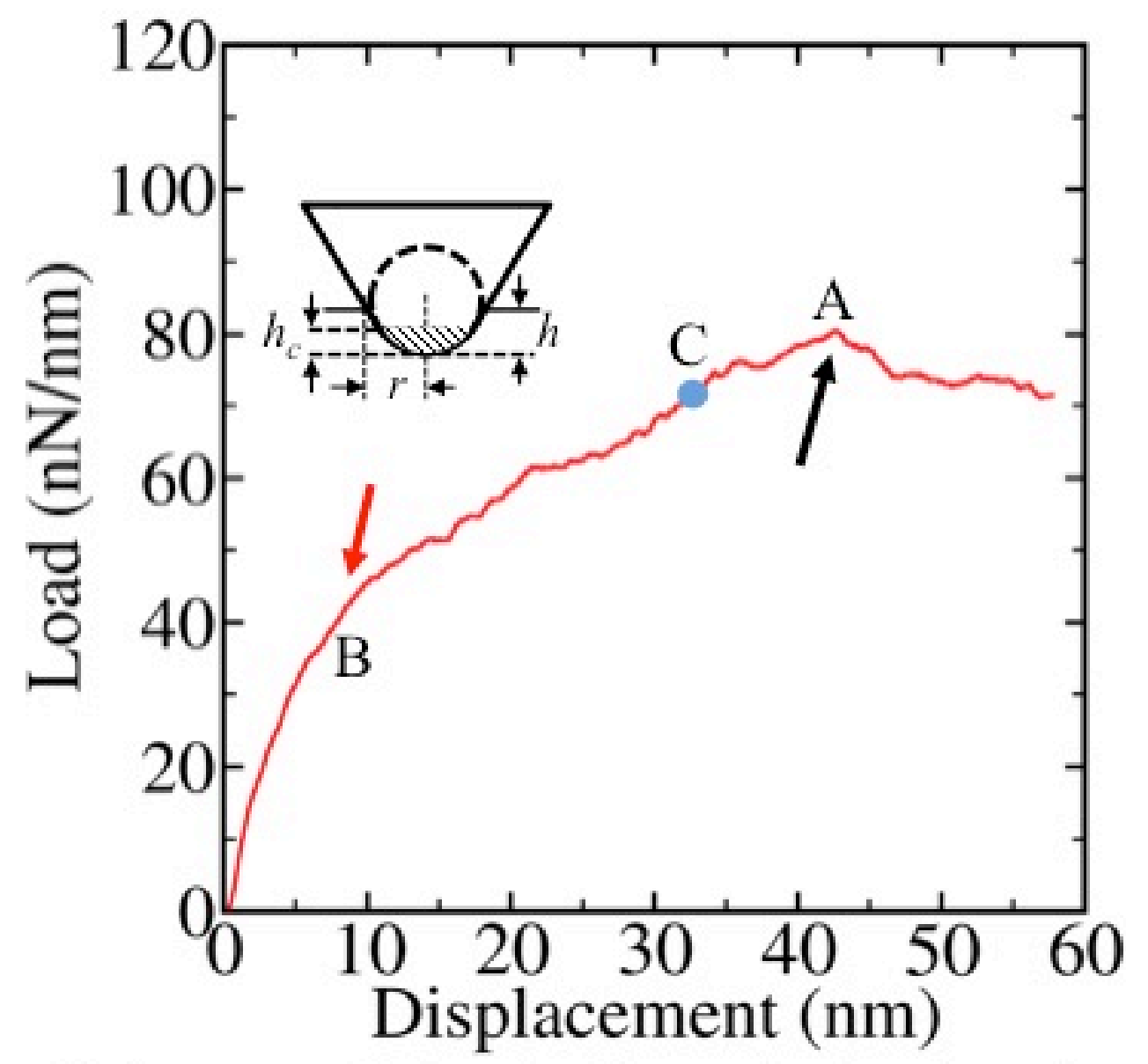

Fig.3 Load versus displacement during loading and unloading phases of indentation. The maximum indenter penetration for all indentation simulations is $\sim 20 \%$ of the sample thickness $(\sim 300 \mathrm{~nm})$. The black arrow (A) denotes the moment when the crack in the sample starts propagating. The red arrow (B) denotes when the wedge part of the indenter makes contact with the sample. The blue $\operatorname{dot}(\mathrm{C})$ is the crack initiation point. $h$ and $r$ in the inset denote the indenter penetration and half of the contact width. $h_{c}$ denotes the critical penetration beyond which the wedge part makes contact with the sample. 


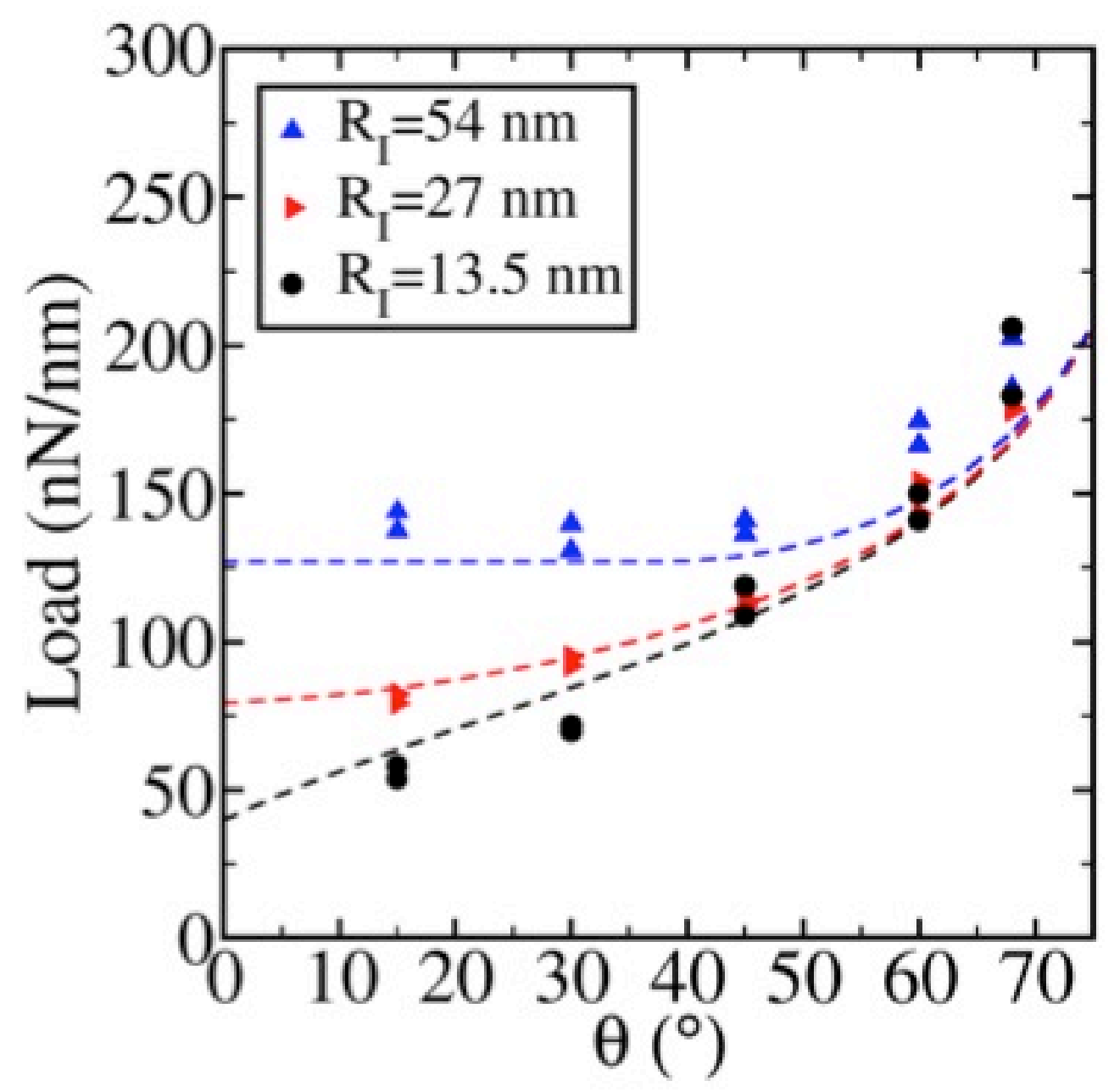

Fig.4 Critical load versus indenter tip angle. The data are extracted from two independent samples. 

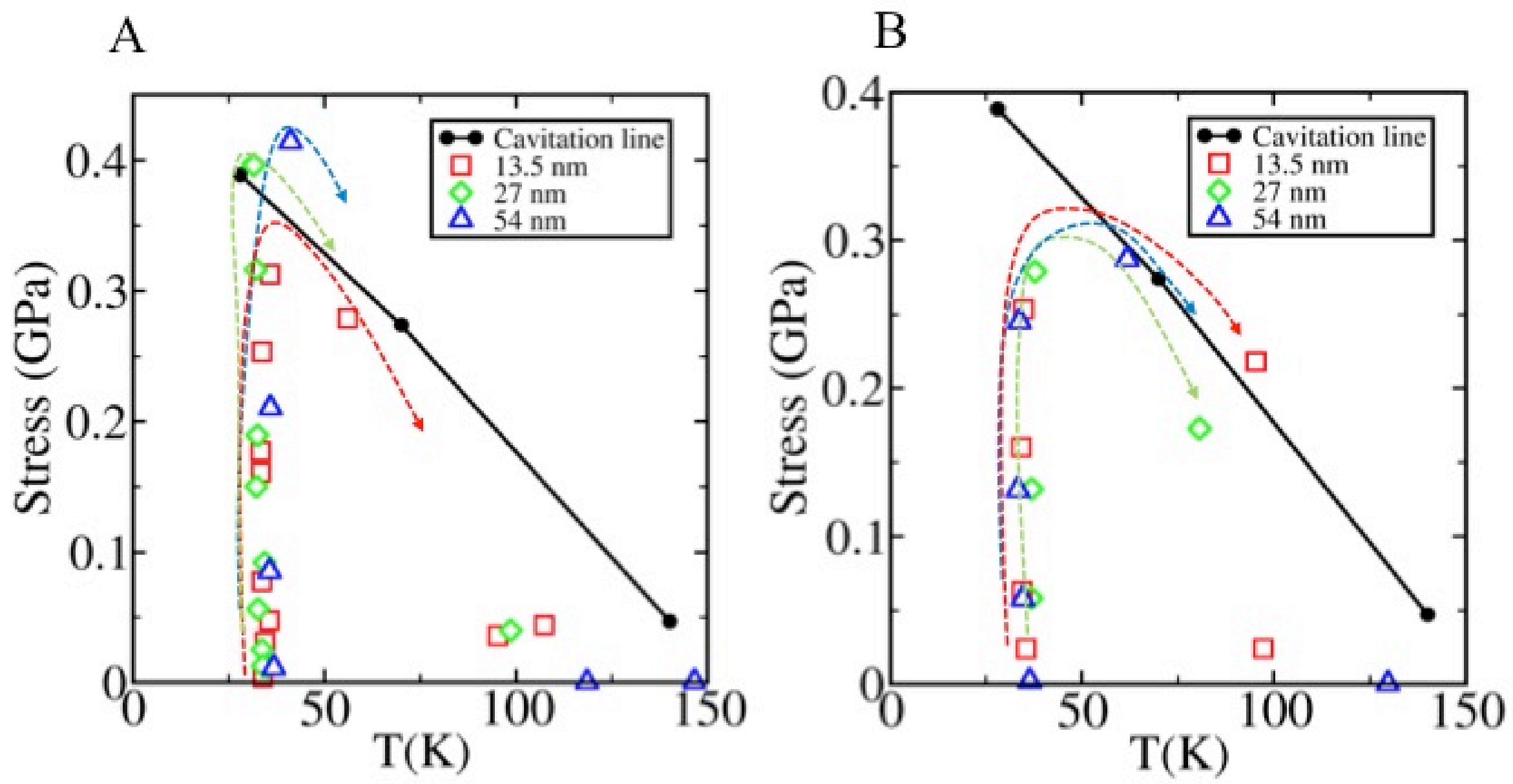

Fig.7 Cavitation map in the normal stress $\left(\sigma_{z z}\right)$ and temperature (T) domain with the thermomechanical state evolution of the cavitation sites during indentation tests. Results from two indenter tip semi-angles $(\theta)$ (left: $30^{\circ}$ and right: $60^{\circ}$ ) and three tip radius $\left(R_{I}\right)$ are included. The cavitation line is measured in Fig. 8. 

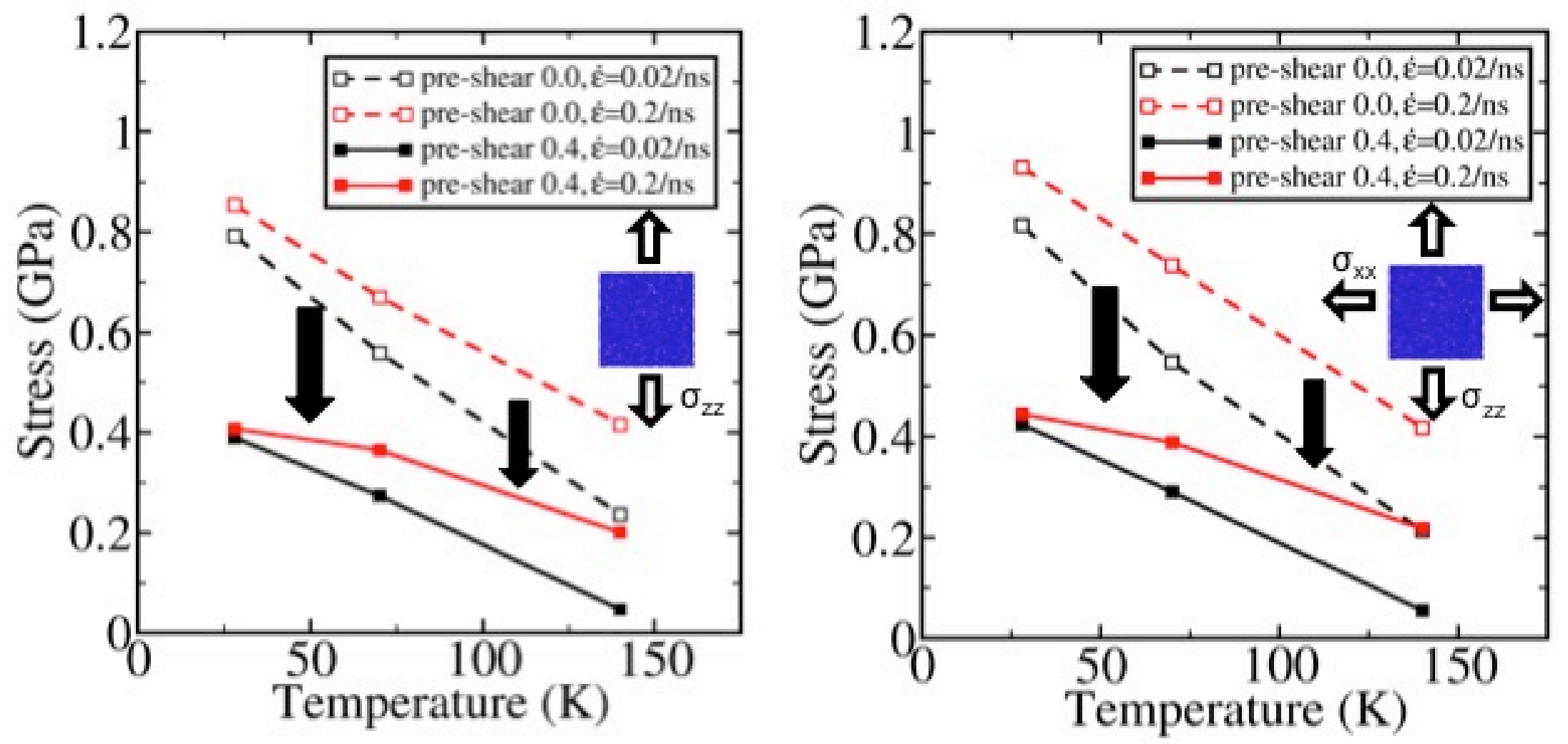

Fig. 8 Thermo-mechanical conditions for cavitation of small samples $\left(\varepsilon_{\mathrm{B}}=0.375\right)$ in the stress and temperature domain. Both uniaxial tension (A) and biaxial tension (B) conditions were used. $\dot{\varepsilon}$ represents either the uniaxial tension strain rate or the biaxial tension strain rate. In both tests, two pre-shears $(0 \%$ and $40 \%)$ were applied to the samples. As can be seen from above, the cavitation stresses from both conditions are quite close to each other. 
Critical macroscopic load

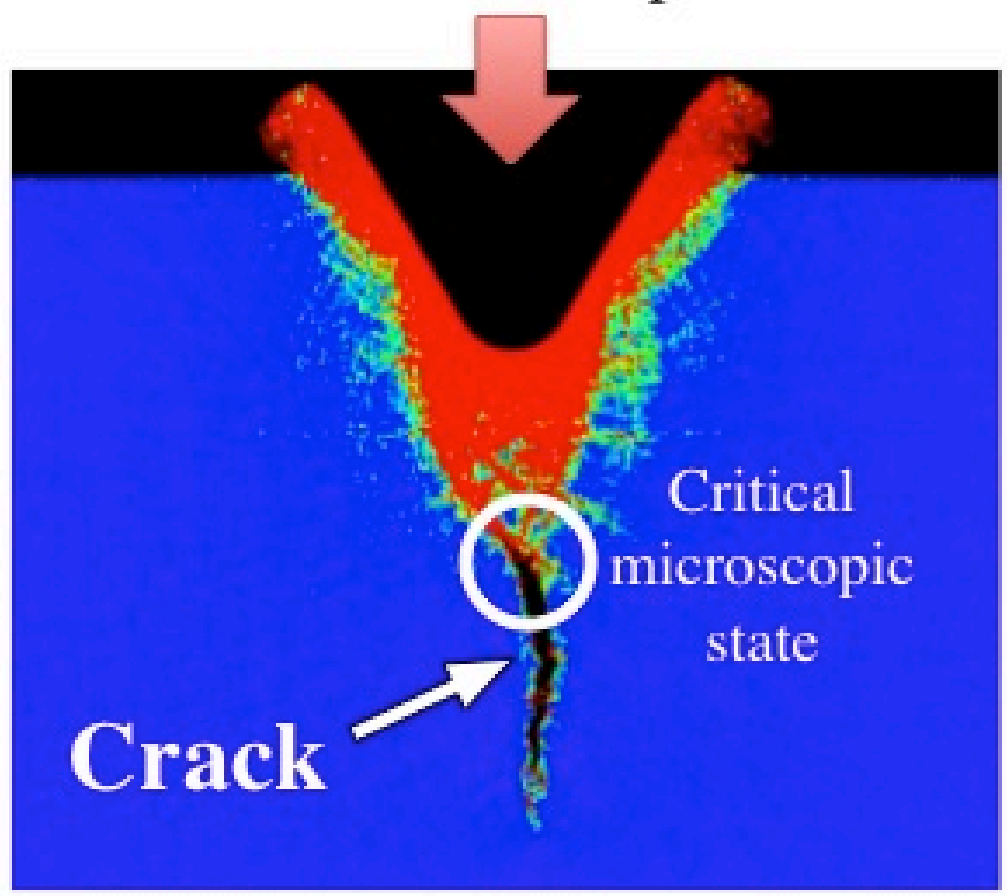

Local shear

$20 \%$

0 